\title{
Rhombomères, code Hox, crête neurale et malformations de la face
}

Gérard Couly

Pierre Coltey

Gérard Chéron

Véronique Abadie

Hélène Martelli

Nicole $M$. Le Douarin

Les recherches réalisées sur l'embryon d'oiseau au moyen des chimères caille-poulet ont montré que les structures faciales et hypobranchiales sont dérivées de la crête neurale. Les cellules issues de cette structure transitoire expriment le même code Hox que celles du rhombomère dont elles sont issues. Ce code est défini par l'expression de gènes à homeobox selon une combinatoire déterminée, caractéristique de chaque rhombomère. L'altération expérimentale du code $H o x$ à certains stades critiques du développement provoque des malformations de la région branchiorhombomérique correspondante. Les fréquentes malformations de la face et du cou en clinique humaine sont interprétées ici à la lumière des découvertes récentes concernant la segmentation de la région branchiorhombomérique et le contrôle de son développement par les gènes sélecteurs des complexes Hox. L'examen des anomalies de la face permet de soupçonner l'existence de malformations concomitantes du cerveau. En effet, les structures faciales étant dérivées essentiellement des crêtes neurales, elles constituent une sorte de "marqueur" de la neurulation et, par conséquent, du développement des niveaux encéphaliques correspondants.

\footnotetext{
ADRESSES

G. Couly: professeur des universités, praticien hospitalier. Service de chirurgie maxillofaciale, plastique et de stomatologie pédiatrique, faculté et hôpital Necker-EnfantsMalades, 149, rue de Sèvres, 75015 Paris, France. P. Coltey: ingénieur d'études Cnrs. Institut d'embryologie cellulaire et moléculaire du Cnrs et du Collège de France, 49bis, avenue de la Belle-Gabrielle, 94736 Nogent-sur-Marne, France. G. Chéron, V. Abadie: pédiatres. H. Martelli : maître de conférences des universités, praticien hospitalier de chirungie pédiatrique. Hôpital Necker-EnfantsMalades, 149, rue de Sèvres, 75015 Paris France. N.M. Le Douarin : professeur au Collège de France, directeur de l'institul d'embryologie cellulaire moléculaire du Cnrs et du Collège de France. Institut d'embryologie cellulaire et moléculaire du Cnrs et du Collège de France, 94736 Nogent-sur-Marne, France.
}

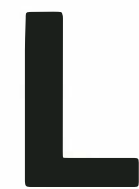

'organisation du corps en segments répétitifs distribués le long de l'axe antéropostérieur est observée chez de nombreux organismes. Il en est ainsi par exemple des vers annélides et des arthropodes (insectes, crustacés, araignées). Chez les vers de terre, à l'exception de la "tête" (ou prostomium) et de l'extrémité post-anale (ou pygidium), l'organisme est fait d'anneaux presque identiques, pourvus chacun d'un ganglion nerveux, de néphridies et de gonades. Le tube digestif est commun à l'ensemble des anneaux, et des nerfs longitudinaux réunissent les ganglions les uns aux autres, con- férant ainsi une certaine unité à l'organisme. Chez les arthropodes, la métamérisation est aussi bien visible, mais les différents segments acquièrent une spécialisation au cours du développement et l'organisation générale du corps est plus complexe.

L’organisation métamérique des vertébrés, bien que plus discrète, a été fréquemment soulignée. Elle est surtout évidente dans les formes primitives, telles que les myxines et les lamproies, dont les fentes branchiales sont distribuées latéralement selon l'axe céphalocaudal en deux rangées régulières. Un reste de la métamérisation du pharynx se mani- 


\section{RÉFÉRENCES}

1. Mccinnis W, Levine MS, Hafen E, Kuroiwa A, Gehring WJ. A conserved DNA sequence in homoeotic genes of the Irosophila Antennapedia and bithorax complexes. Nature 1984; 308: 428-33.

2. Mc(innis W, Garber RL, Wirz J, Kuroiwa A, (jehring WJ. A homologous protein-coding sequence in Drosophila homeotic genes and its conservation in other metazoans. Cell 1984; 37 : 403-8.

3. Scott MP, Weiner AJ. Structural relationships among genes that control development : sequence homology between the Antennapedia, Ultrabithorax, and Jushi tarazu loci of Irosophila. Proc Natl Acad Sci USA $1984 ; 81: 4115-9$.

4. Lewis EB. A gene complex controlling segmentation in Irosophila. Nature 1978; 276: $565-70$.

5. Carrasco AE, McGinnis W, Gehring WJ, de Robertis EM. Cloning of a $X$. laevis gene expressed during early embryogenesis coding for a peptide region homologous to Drosophila homeotic genes. Cell $1984 ; 37$ : 409-14.

6. McGinnis W, Hart CP, Gehring WJ, Ruddle FH. Molecular cloning and chromosome mapping of a mouse DNA sequence homologous to homeotic genes of Irosophila. Cell 1984; 38: 675-80.

7. De Robertis EM, Oliver G, Wright CV. Les gènes à homeobox et l'organisation du corps. Pour la Science 1990; 155: 50-6.

8. Kessel M, Gruss P. Murine developmental control genes. Science $1990 ; 249: 374-9$.

9. Goodrich ES. On the development of segments of the head in Scyllium. J Microse Sci $1918 ; 63: 1-30$.

10. Orr H. Contribution to the embryology of the lizard. J Morphol 1887; 1: 311-72.

11. Vaage $S$. The segmentation of the primitive neural tube in chick embryo (C;allus domesticus). Adv Anat Embryol (eell Biol $1969 ; 41: 1-88$.

12. Fraser S, Keynes R, Lumsden A. Segmentation in the chick embryo hindbrain is defined by cell lineage restrictions. Nature 1990 ; 344: 431-5.

13. Le Lièvre CS, Le Douarin NM Mesenchymal derivatives of the neural crest : analysis of chimaeric quail and chick embryos. J Embryol Exp Morphol 1975; 34 : $125-54$. feste che $z$ l'embryon de tous les vertébrés, y compris les mammifères, dans des arcs branchiaux. De plus, le mésoderme paraxial embryonnaire est organisé en somites qui fournissent ensuite les vertèbres, éléments métamériques du squelette axial. Enfin, les nerfs et les ganglions rachidiens sont aussi organisés métamériquement le long de la moelle épinière.

Les progrès de la biologie moléculaire ont ouvert des perspectives nouvelles dans l'étude du dévelop-

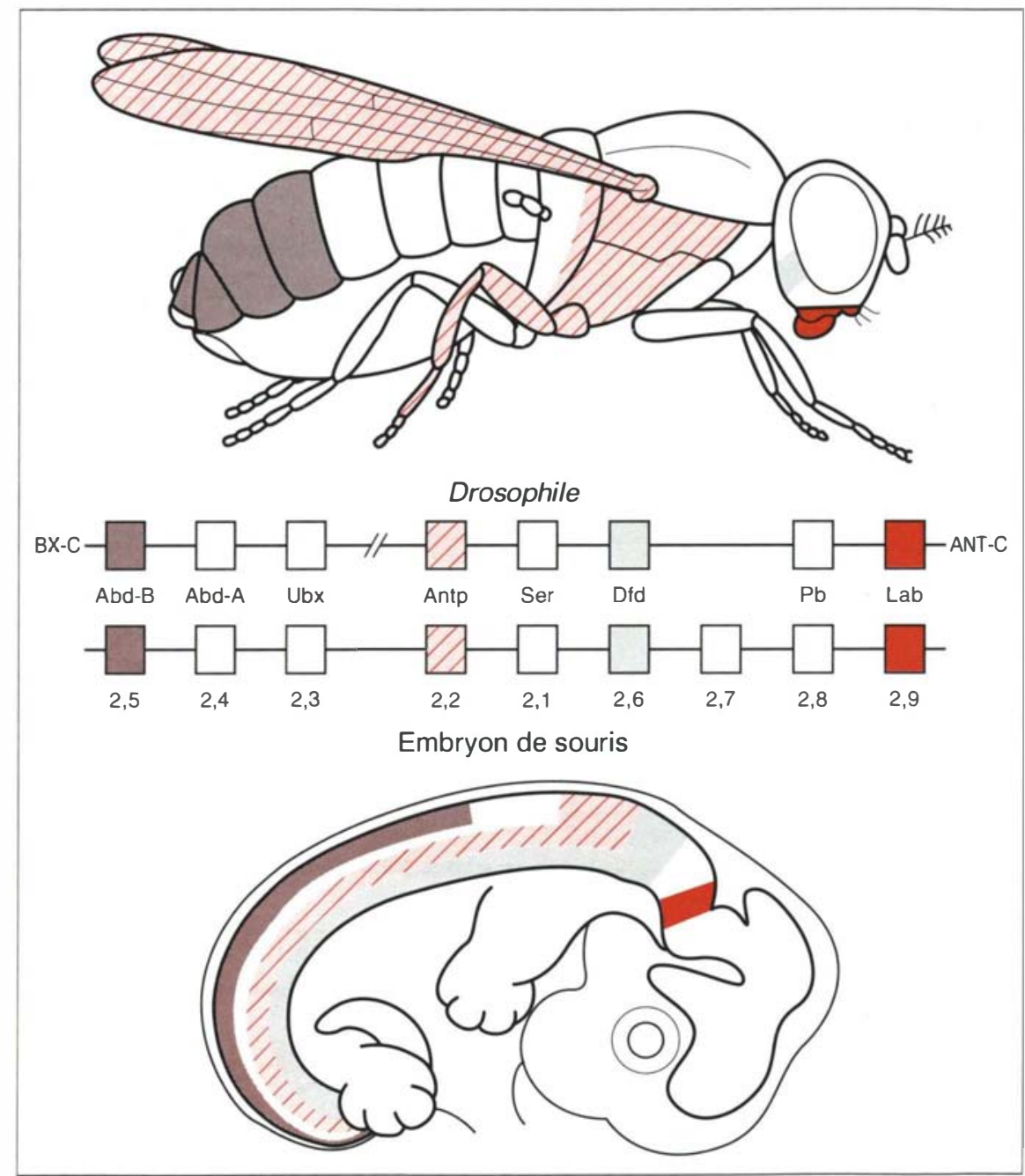

Figure 1. Les gènes à homeobox du complexe HOM-C de la drosophile sont distribués sur un seul chromosome et s'expriment chez la larve à des niveaux et à des stades du développement correspondant à leur position sur le chromosome. Les gènes exprimés dans la tête (Lab, Pb) sont situés en $3^{\prime}$ sur la molécule d'ADN et sont les premiers à être transcrits. Les gènes des complexes Hox de la souris sont répartis en quatre groupes sur quatre chromosomes différents. Ils résultent probablement de deux duplications successives d'un complexe homologue à celui de la drosophile. Le même principe de colinéarité contrôle leur expression selon l'axe antéropostérieur de l'embryon. La limite antérieure d'expression des gènes Hox se situe entre les rhombomères 2 et 3. (D'après [7] avec permission).

pement embryonnaire. Des mutations affectant le développement chez la drosophile ont conduit à la responsables de la mise en place du plan d'organisation de la future l'embryogenèse. Il s'agit de gènes codant pour des protéines régulatrices capables de contrôler le foncleur capacié autres gènes grace déterminées de l'ADN.

Il en est ainsi d'une famille de 
gènes possédant une séquence de 180 paires de bases appelée homeobox qui code pour un homéodomaine responsable de l'activité régulatrice de la protéine qui le porte. Le clonage du premier gène à homeobox, responsable de la mutation Antennapedia chez la drosophile, a eu lieu indépendamment dans deux laboratoires au début des années 1980 [1-3]. La mutation Antennapedia appartient aux mutations dites " homéotiques ", caractérisées par la transformation d'une structure en une autre. Ainsi, dans la mutation Antennapedia, l'antenne est remplacée par une patte.

Une série de mutations homéotiques ont été décrites chez la drosophile et l'analyse génétique qu'en a réalisée Lewis [4] a révélé qu'elles correspondent à des gènes distribués linéairement sur un même chromosome formant ainsi les complexes Ultrabithorax (Ubx) et Antennapedia (Antp) (ils portent aussi le nom de complexe $H(M-C)$. La révélation par Gehring, de Robertis et al. en 1984
[5] que des gènes à homeobox existent aussi chez les vertébrés a ouvert la voie à une importante série de travaux. Le premier gène à homeobox de vertébré a été découvert chez le xénope. Peu après, on clonait un gène à homeobox chez la souris [6], suivi par de nombreux autres dont on établissait la localisation chromosomique (pour revues, voir de Robertis [7] et Kessel et Gruss [8] ) (figure 1). Les gènes à homeobox dont la séquence nucléotidique présente des homologies importantes

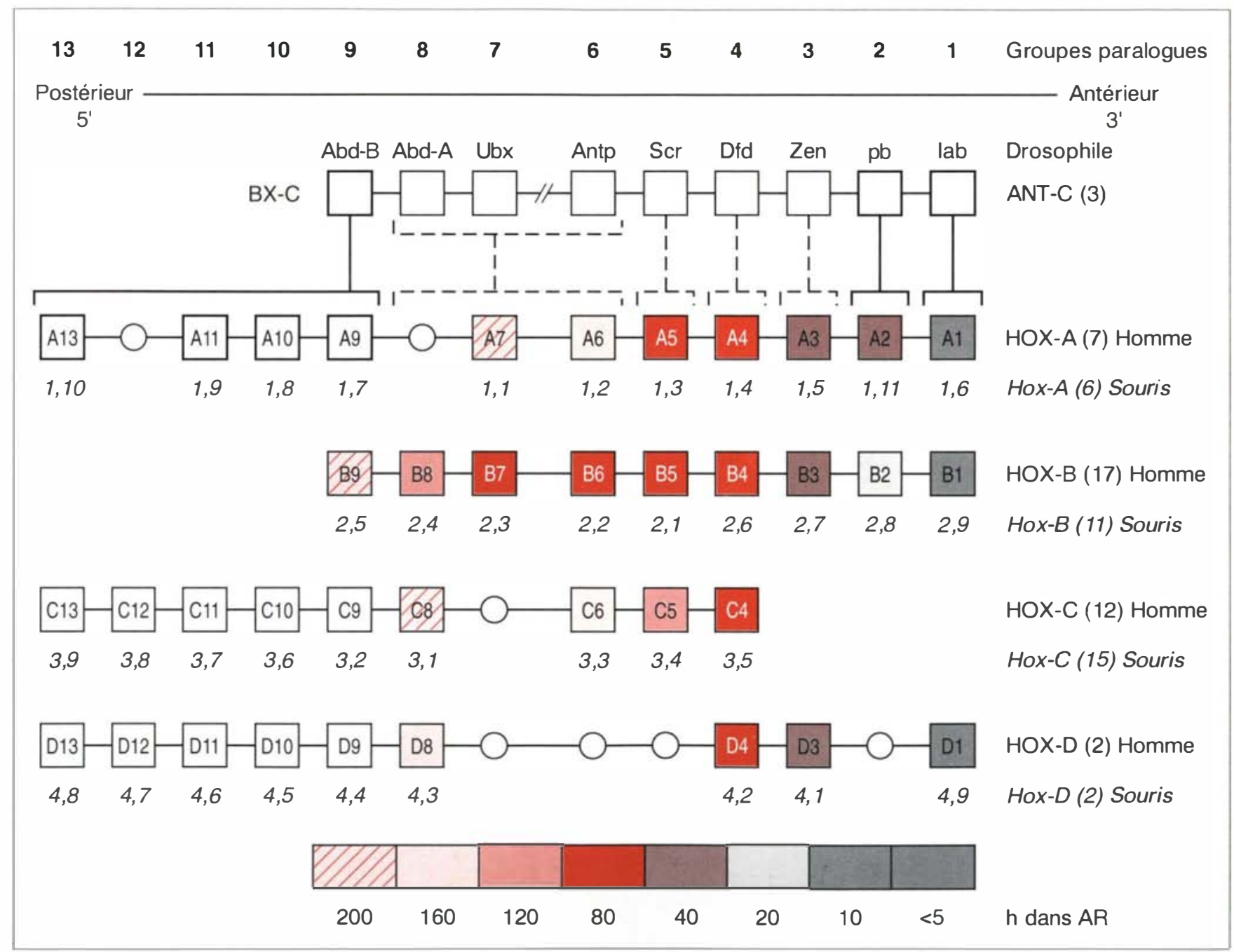

Figure 2. Les gènes du complexe HOM-C de la drosophile et les gènes des quatre clusters HOX chez I'homme et la souris, selon la nouvelle nomenclature [33]. Les gènes présentant la plus grande homologie entre eux sont les gènes "paralogues" situés au même niveau dans chaque groupe (A4, B4, C4, D4). L'expression de certains gènes Hox est activée par l'acide rétinoïque (AR). L'activation décroît des gènes les plus antérieurs (A1, B1, C1, D1) à ceux qui sont exprimés le plus caudalement, ces derniers pouvant être réprimés par l'acide rétinoïque. Les couleurs indiquent la sensibilité décroissante des gènes à l'acide rétinoïque en fonction de leur position sur le chromosome. (Modifié d'après [34, 36]). h dans $R$ : durée en heures d'exposition des cellules à l'acide rétinoïque. Les cercles blancs correspondent à des gènes présomptifs non encore isolés. Les nombres entre parenthèses correspondent au chromosome sur lequel le groupe de gènes Hox correspondant se trouve chez l'homme et la souris. 


\section{RÉFÉRENCES}

14. Hunt P, Krumlauf R. Hox codes and positional specification in vertebrate embryonic axes. Annu Rev Cell Biol 1992; 8: $227-56$

15. Couly (;, Le Douarin NM. Head morphogenesis in embronic avian chimeras evidence for a segmental pattern in the ectoderm corresponding to the neuromeres. Development 1990; 108: 543-58.

16. Lohnes I, Kastner P, Dierich A, Mark M, LeMeur M, Chambon P. Function of retinoic acid receptor- $\gamma$ in the mouse. Cell $1993 ; 73: 643-58$

17. Colberg-Poley AM, Voss SD, Chow dhury K, Cruss P. Structural analysis of murine genes containing homeobox sequences and their expression in embryonal carcinoma cells. Nature $1985 ; 314$ 713-8.

18. Breier (;) Bucan M, Francke U Colberg-Poley AM, Gruss P. Sequential expression of murine homeobox genes during F9 EC, cell differentiation. $E M B C$ $1986 ; 5: 2209-15$.

19. Simeone A, Acampora D, Arcioni L, Andrews PW', Boncinelli E, Mavilio F. Sequential activation of Hox2 homeobox genes by retinoic acid in human embryonal carcinoma cells. Nature 1990; 346 763-6.

20. Kessel M, Gruss P. Homeotic transformations of murine vertebrae and concomitant alteration of Hox codes induced by retinoic acid. Cell 1991; 67 : 89-104

21. Kessel M, Balling R, Gruss P. Variations of cervical vertebrae after expression of a Hox-1.1 transgene in mice. Cell 1990;61 301-8.

22. Chisaka O, Capecchi MR. Regionally restricted developmental defects resulting from targeted disruption of the mouse homeobox gene Hox-1.5. Nature 1991 ; 350: 473-9

23. Lufkin T, Dierich A, LeMeur M, Mark M, Chambon P. Disruption of the Hox-1.6 homeobox gene results in defects in a region corresponding to its rostral domain of expression. Cell 1991; 66: 1105-19.

24. Chisaka O, Musci TS, Capecchi MR Developmental defects of the ear, cranial nerves and hindbrain resulting from targeted disruption of the mouse homeobox gene Hox-1.6. Nature $1992 ; 355$ : 516-20.

25. Lufkin T, Mark M, Hart C.P, Dolle P. LeMeur M, Chambon P. Homeotic transformation of the occipital bones of the skull by ectopic expression of a homeobox gene. Nature 1992; 359: 835-41. avec celle des gènes des complexes $A n t p$ et $U b x$ sont au nombre de 38 chez les mammifères. Ils sont disposés en quatre groupes répartis sur quatre chromosomes différents et résultent de duplications d'un groupe ancestral dont descend aussi celui de la drosophile. La distribution des gènes le long de chaque chromosome est la même que dans le complexe HOM-C de la drosophile, de sorte qu'on peut distinguer dans chacun des quatre groupes des gènes dits "paralogues" qui présentent entre eux de forts niveaux d'homologie (figure 2).

Les gènes qui, chez la drosophile, contrôlent le développement embryonnaire sont nombreux; ceux du complexe HOM-C interviennent en aval d'une cascade où d'autres gènes régulateurs sont responsables de la détermination des axes de polarité antéropostérieure et dorso- ventrale et de la mise en place des segments de la mouche. Les gènes du complexe $H O M-C$ sont particulièrement affectés à la détermination de l'identité segmentaire. Une particularité générale de tous les gènes "sélecteurs " qui contrôlent le développement est qu'ils possèdent une ou plusieurs séquences nucléotidiques codant pour un domaine protéique capable de se lier à l'ADN. L'homeobox est l'un de ceux-ci; d'autres motifs tels que la pair box ou les "doigts de zinc" confèrent aussi aux protéines qui les contiennent le rôle de facteurs de transcription.

La découverte des gènes à homeobox chez les vertébrés a été suivie de l'étude de leur expression spatiotemporelle au cours du développement. Les gènes des quatre groupes $H O X$ $A, B, C$ et $D$ sont exprimés transitoirement dans le tube neural,

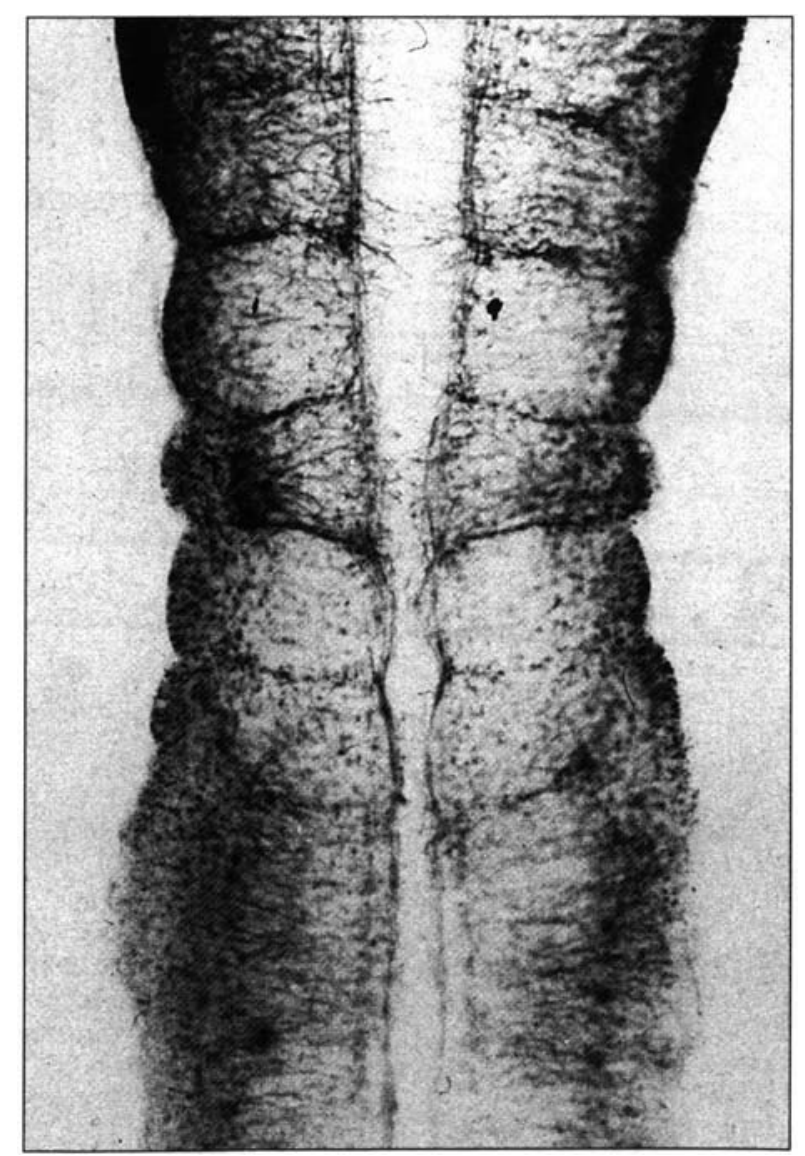

Figure 3. Le rhombencéphale d'un embryon de poulet au stade 16 de Hamburger et Hamilton [35]. La délimitation des segments est soulignée par une coloration in toto par un anticorps dirigé contre les protéines des neurofilaments. (Reproduit d'après [36] avec permission). 
les somites et les membres, selon une séquence spatio-temporelle déterminée qui pourrait être le sup port d'une information positionnelle le long de l'axe céphalocaudal de l'embryon et de l'axe proximodistal des membres. Comme chez la drosophile, la séquence et la limite rostrale d'expression de chaque gène d'un groupe donné obéit au principe de colinéarité selon lequel les gènes situés en 3' du complexe sont ceux dont l'expression est, à la fois, la plus précoce et la plus rostrale.

L'expression spatio-temporelle des gènes des complexes Hox au niveau du cerveau postérieur et du pharynx a été particulièrement bien étudiée et suggère qu'ils pourraient jouer un rôle dans la détermination régionale de cette partie de l'organisme. Ces observations ont dirigé à nouveau l'attention des embryologistes sur l'idée ancienne d'une métamé- risation primitive de l'ébauche neurale des vertébrés, qui présiderait à l'organisation fonctionnelle ultérieure du système nerveux.

\section{La segmentation du système nerveux des vertébrés*}

Comme cela a été mentionné plus haut, la segmentation la plus évidente dans toutes les formes de vertébrés concerne le pharynx et la division du mésoderme en somites. Goodrich en 1918 [9] concevait un plan général d'organisation des vertébrés dans lequel il liait la métamérisation du mésoderme à celle du système nerveux qui est, selon lui, organisé en segments dans toute sa longueur. De chacun des "segments " nerveux, ou neuromères, sont issus des nerfs pairs qui inner- vent les muscles correspondants. Les arcs branchiaux appartiennent aux "segments céphaliques".

En fait, l'existence de neuromères dans la moelle épinière n'a pas été confirmée. Les motoneurones dont sont issus les nerfs rachidiens ventraux sont distribués d'une manière uniforme le long de l'axe neural, en tout cas chez les vertébrés supérieurs.

On ne peut considérer qu'on a affaire à un vrai métamère que s'il existe des relations de lignage entre les cellules qui le constituent. Cela implique qu'il n'y a pas de mélanges de cellules d'un métamère à l'autre et qu'ils doivent être séparés par des cloisons que les cellules ne franchissent pas. De tels métamères ont été décrits dans le rhombencéphale par Orr [10] puis par Vaage [11] chez l'embryon de poulet (figure 3). On leur a donné le nom

Tableau

DÉRIVÉS DE LA CRÊTE NEURALE AU NIVEAU CÉPHALIQUE (D'après [34])

Dérivés mésectodermiques céphaliques

Cellules nerveuses

- Neuroblastes bipolaires (somato-sensibles)
- Squelette facial et de la voûte du crâne (os, cartilages)

- Odontoblastes (dents)

- Paroi arcs aortiques (IIIe, IVe, Vle)

- Derme de la face et du cou, muscles horripilateurs et calvarium

- Conjonctif, thymus, parathyroïdes, thyroïde, glandes salivaires et lacrymales, hypophyse

- Participation musculature faciale

- Sclérotique, muscles ciliaires, choroïde

- Méninges prosencéphaliques

- Ganglions rachidiens

- Ganglions des nerfs crâniens (V, VII, X, XI)

- Neuroblastes multipolaires

Cellules des ganglions du système nerveux autonome

- à phénotype cholinergique (parasympathique bulbaire)

- à phénotype adrénergique (sympathique)

\section{Cellules gliales}

\section{Cellules pigmentaires}

\section{Cellules endocriniennes} (phénotypes peptidiques) et para-endocriniennes
- de Schwann

- Satellites des ganglions du système nerveux périphérique

- Glie entérique

- Cellules C à calcitonine (thyroïde)

- Cellule I et II du corps carotidien

- Glande médullo-surrénale et para-ganglions.

\footnotetext{
* Sur le même sujet, voir aussi lexique embryologie médecine/sciences, la morphogenèse du cerveau postérieur, m/s $n^{\circ} 8 / 9$, vol. 9, p. 975. $\mathrm{m} / \mathrm{s} n^{\circ} 2$ vol. 10 , fériner 94
} 


\section{RÉFÉRENCES}

26. Couly G, Aicardi J. Anomalies associées de la face et de l'encéphale chez le nourrisson et l'enfant. Arch Fr Pediatr 1988; 45 : 99-104.

27. Couly G. Malformations de la face et du cou. Stomatologie Odontologie. Encycl Med Chir (Paris France) : 22066 A5012P, 1992.

28. Couly G, Ayer-Le Lièvre C. Malformations latéro-faciales (neurocristopathies maxillo-mandibulaires) associées à des anomalies du tronc cérébral). Rev Stomatol Chir Maxillofac 1983; 84: 254-63.

29.Aleksic S, Budzilovich G, Choy A, Reuben R, Randt C, Finegold M, McCarthy J, Converse J, Feigin I. Congenital ophtalmoplegia in oculoauriculovertebral dysplasiahemifacial microsomia (Goldenhar-Gorlin syndrome). A clinicopathologic study and review of the literature. Neurology 1976; 26 : 638-44.

30. Couly G, Lagrue A, Griscelli C. Le syndrome de Di George, neurocristopathie rhombencéphalique. Rev Stomatol Chir Maxillofac 1983; 84 : 103-8.

31. Couly G, Chéron $G$, de Blic J, Despres C, Cloup M, Hubert P. Le syndrome de Pierre Robin. Classification et nouvelle approche thérapeutique. Arch $\mathrm{Fr}$ Pediatr 1988 ; 45 : 553-9.

32. Kirby ML, Gale TF, Stewart DE. Neu ral crest cells contribute to normal aorticopulmonary septation. Science 1983 ; 220 : 1059-61.

33. Abadie V, Chéron G, Couly G. Syndrome néonatal de dysfonctionnement du tronc cérébral. Arch Fr Pediatr 1993 ; 347-52.

34. Scott MP. Vertebrate homeobox gene nomenclature. Cell 1992; 71 : 551-3.

35. Hamburger V, Hamilton HL. A series of normal stages in the development of the chick embryo. J Morphol 1951 ; 88 : 49-92.

36. Lumsden A. The development and significance of hindbrain segmentation. Semin Dev Biol 1990; 1: 117-25.

37. Lumsden A, Keynes R. Segmental patterns of neuronal development in the chick hindbrain. Nature 1989; 337: 424-8.

38. Hunt P, Gulisano M, Cook M, Sham MH, Faiella A, Wilkinson D, Boncinelli E, Krumlauf R. A distinct Hox code for the branchial region of the vertebrate head. Nature 1991 ; 353: 861-4.

39. Mavilio F. Review : regulation of vertebrate homeobox-containing genes by morphogens. Eur J Biochem 1993; 212: 273-88. de rhombomères, mais leur existence, seulement transitoire, a été mise en doute et même oubliée pendant plus de vingt ans. L'observation que plusieurs des gènes des complexes Hox ont une limite antérieure d'expression correspondant à celle qui sépare deux rhombomères successifs a ressuscité l'intérêt pour ces structures et leur signification. Fraser, Keynes et Lumsden [12] ont montré par d'élégantes expériences d'injection intracellulaire de colorants vitaux que les cellules du tube neural ventral rhombencéphalique ne franchissent plus la barrière virtuelle située entre deux rhombomères à partir du moment où ceux-ci sont reconnaissables chez l'embryon de poulet. En revanche, si l'injection est faite avant la délimitation des segments, le clone dérivé de la cel- lule injectée s'étend sur au moins deux rhombomères voisins. Fraser et al. ont ainsi pu tracer l'origine des noyaux dont sont issus les nerfs crâniens associés au rhombencéphale, et montrer que chaque nerf correspond à un noyau situé dans deux rhombomères contigus (figure 4A). La région rhombencéphalique des vésicules cérébrales est particulièrement importante dans la genèse des structures faciales. Dès les années 1970, l'application de la méthode de marquage, par la construction de chimères caille-poulet, avait permis d'élucider le devenir des cellules de la crête neurale dans le modèle aviaire et de montrer que la totalité du squelette et du derme de la face et de la région hypobranchiale provient de la crête neurale pro-, mes- et rhombencéphali-

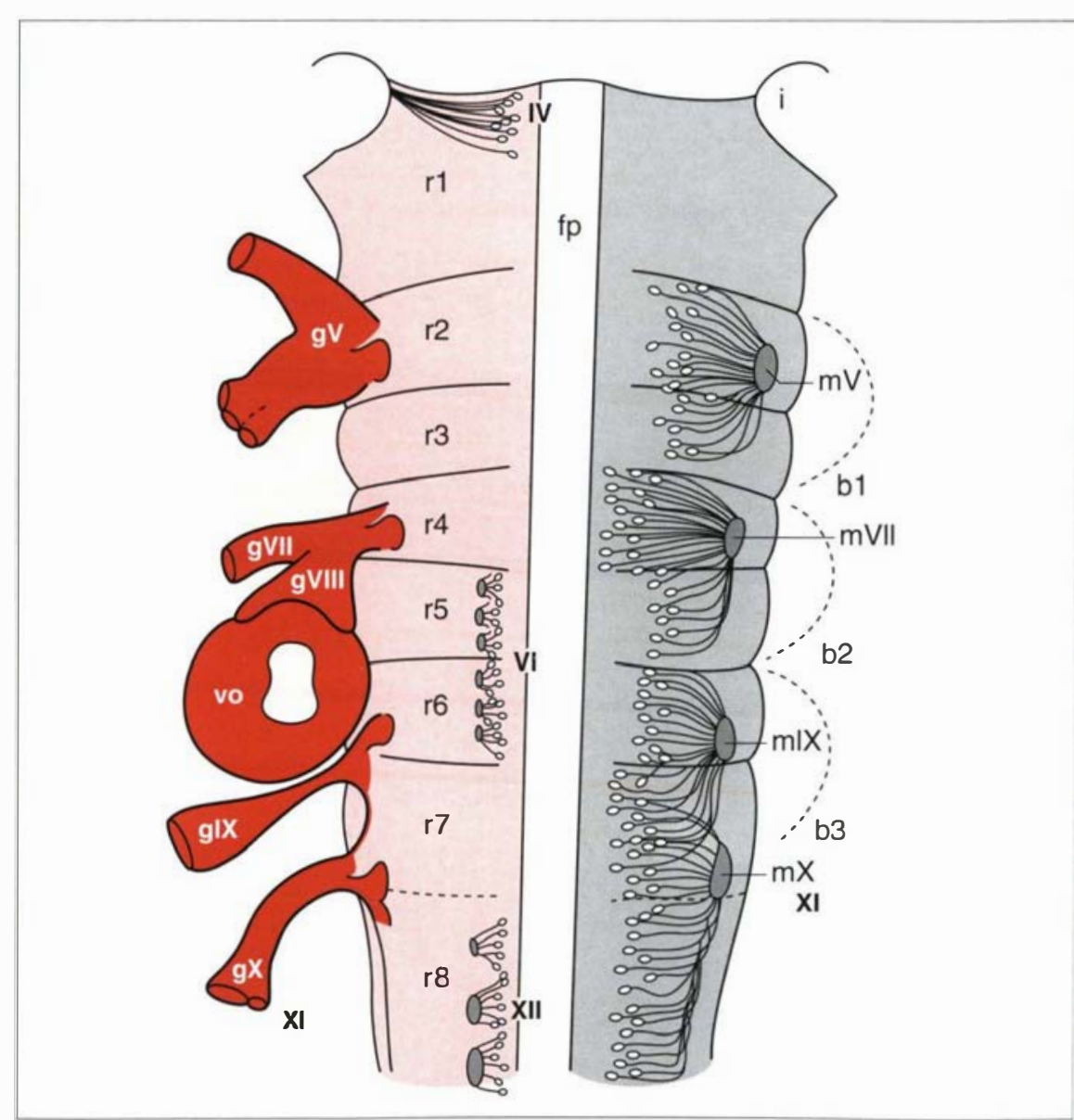

Figure 4. A. Schéma représentant à gauche l'origine segmentaire des nerfs crâniens gV-gXI (r1-r8: rhombomères 1 à 8) et à droite les corps cellulaires des neurones moteurs projetant des fibres dans les nerfs crâniens $m \boldsymbol{V}$ - $\boldsymbol{m X I}$. vo = vésicule otique. (D'après [37] avec permission). 
que [13] (Tableau I). La région rhombencéphalique elle-même fournit le mésenchyme des arcs brain chiaux (mandibule et région hypobranchiale).

L'expression des gènes des complexes Hox est caractérisée par l'association, au niveau de chaque segment et à un moment déterminé de l'ontogenèse, d'une combinaison unique de produits géniques définissant un code (code Hox) pour lequel on pouvait soupçonner un rôle dans la spécification de chacun de ces segments (figure 4B). Les travaux des laboratoires de Hunt, Krumlauf et Wilkinson, réalisés sur la souris, ont été essentiels dans la mise en évidence de l'expression temporo-spatiale des gènes Hox dans le cerveau postérieur et dans les cellules de la crête neurale qui en sont issues. Celles-ci expriment apparemment les mêmes gènes que les segments rhombomériques dont elles proviennent (figure 4B) [14].

Nos propres travaux, fondés sur le marquage des cellules de la crête neurale ou de l'ectoderme superficiel des arcs branchiaux par la construction de chimères caille-poulet ont montré que chaque arc branchial, ainsi que son innervation, correspondent à deux rhombomères contigus, définissant ainsi des unités segmentaires ectodermiques, les "ectomères" (figure 5) [15]. Selon les études réalisées chez la souris, les gènes du complexe Hox-2 sont d'abord exprimés dans les cellules de la crête neurale en migration, et, dans un second temps seulement,

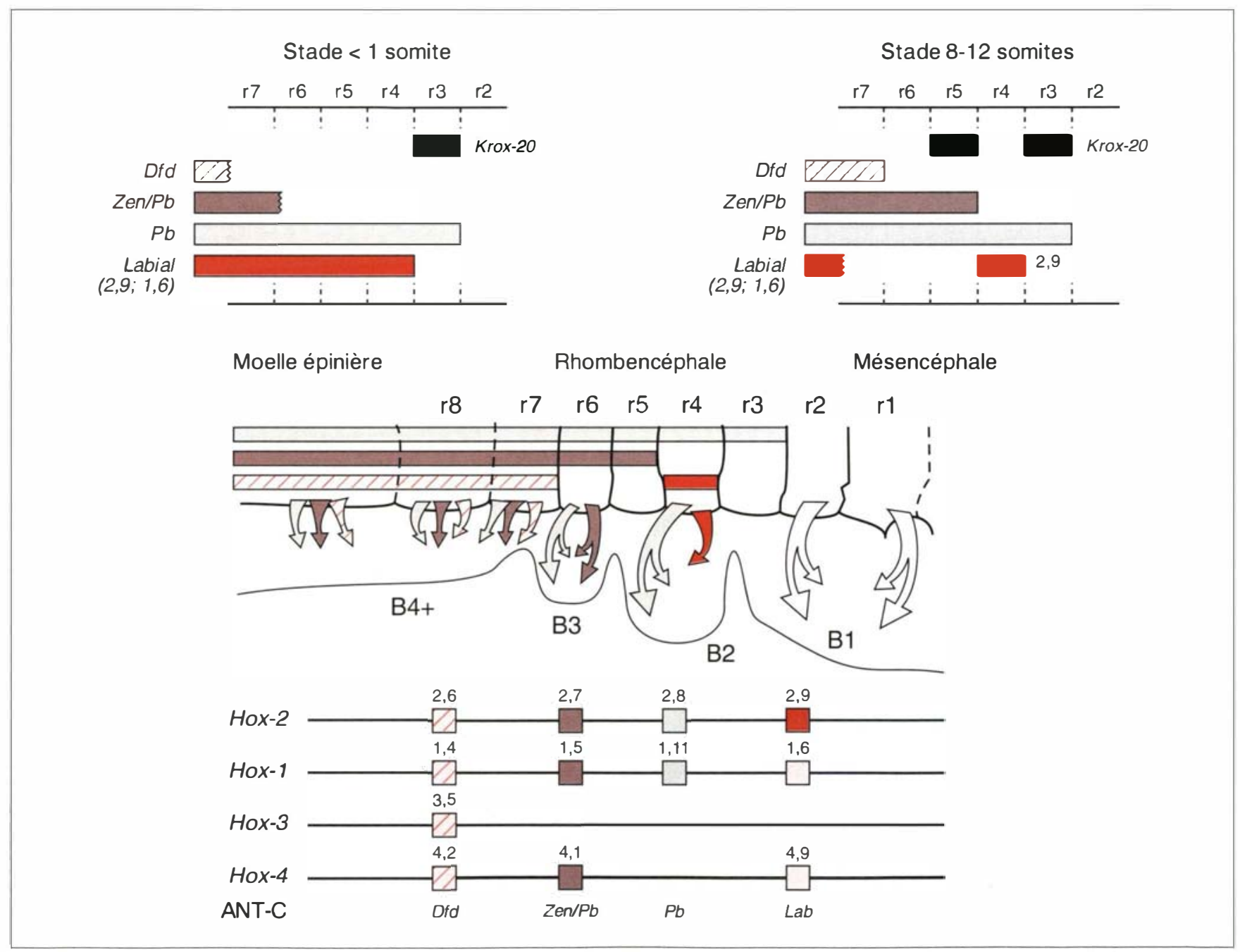

Figure 4. B. Niveaux d'expression des gènes des quatre groupes Hox dans le rhombencéphale et les crêtes neurales qui en sont issues. Avant l'apparition de rhombomères identifiables (stade < 1 somite), les limites supposées des rhombomères sont indiquées en pointillé. Les gènes correspondant aux domaines d'expression des sous-familles sont montrés au bas du schéma avec les mêmes couleurs. Les gènes qui n'ont pas atteint leur limite de migration antérieure ont une limite antérieure en ligne brisée. B1 à B4: arc branchial 1 à 4 . L'expression des différents gènes Hox à différents niveaux du tronc cérébral et des arcs branchiaux varie selon le niveau considéré. Un code Hox est ainsi engendré. II paraît déterminant dans la spécification de cette région de l'embryon tant au plan de l'innervation que de la morphogenèse branchiofaciale. La migration de la crête neurale, indiquée par les flèches de couleur, a transféré un code Hox combinatoire aux ganglions des nerfs craniens et aux arcs branchiaux. (Reproduit d'après [38] avec permission). 
dans celles de l'ectoderme superficiel correspondant, d'où l'idée que la spécification du niveau transversal est d'abord établie dans le neurectoderme, un transfert d'information de la crête neurale à l'ectoderme superficiel se produisant ensuite.

\section{Implication du " code Hox" dans la morphogenèse branchio-cervicale}

Les gènes des complexes Hox ne se situent pas à l'origine de la cascade d'événements qui déterminent la segmentation du cerveau postérieur et des arcs branchiaux. On sait qu'ils sont soumis eux-mêmes à une régulation relayée par l'acide rétinoïque. L'acide rétinoïque est un morphogène dont l'importance a été démontrée par de nombreux travaux anciens de tératogenèse induite par la carence ou l'excès de vitamine $A$, ainsi que par des découvertes récentes sur son mode d'action au niveau moléculaire. Dans ce domaine, les laboratoires de Chambon à Strasbourg (France) et Evans en Californie (USA) ont joué un rôle essentiel en clonant les gènes des récepteurs de l'acide rétinoïque. Comme ceux des stéroïdes, les récepteurs de l'acide rétinoïque sont capables de se lier à l'ADN et jouent le rôle de facteurs de transcription, contrôlant ainsi l'activité de gènes cibles. La distribution des récepteurs de l'acide rétinoïque obéit à un " patron " spatio-temporel précis au cours du développement. La mutation ciblée de certains de ces récepteurs, réalisée récemment par le groupe de Strasbourg chez la souris [16], produit des anomalies comparables à celles qui résultent de la carence en vitamine $A$ au cours du développement fotal.

La notion qu'il existe une interaction entre l'acide rétinoïque et les gènes Hox résulte de l'observation émanant du laboratoire de Gruss montrant que l'acide rétinoïque active certains gènes $H o x$ dans des cellules de tératocarcinome en cours de différenciation [17]. L'importance et la dynamique de cette activation varient selon le gène consi- cloné les gènes des quatre complexes Hox humains, ont réalisé une étude systématique de ce phénomène et montré que la règle de colinéarité s'applique aussi à l'induction des gènes Hox par l'acide rétinoïque : les gènes en 3' sont activés les premiers et l'induction se propage ensuite de 3' en 5'. Certains gènes, situés en aval de chaque complexe, sont inhibés et non pas activés par l'acide rétinoïque [19] (figure 2).

Ces découvertes ont amené Kessel et Gruss [20] à tenter de perturber l'expression des gènes $H o x$ au cours du développement de la souris en injectant à la mère, à 7,5 jours de gestation, des doses tératogènes d'acide rétinoïque. Il en résulte une modification du " code Hox" notamment dans la région branchiocervicale, où l'on observe une transformation homéotique des premières vertèbres cervicales.

Ce résultat suggérait fortement que les gènes régulateurs des complexes Hox jouent un rôle dans l'organisation du cerveau postérieur et des régions squelettiques et branchiales qui lui sont associées. Ce rôle a été confirmé par l'expérience de Kessel, Balling et Gruss [21] dans laquelle le gène Hox 1-1 (maintenant appelé Hox A7), placé sous le contrôle d'un promoteur particulièrement efficace, est injecté dans l'œuf de souris. Une telle transgénèse modifie encore le code Hox et provoque des anomalies des vertèbres cervicales et notamment la formation d'un proatlas et d'un atlas muni d'un corps vertébral. Enfin, les mutations ciblées des gènes Hox 1-5 (Hox A3) ([22], (m/s $n^{\circ} 6$, vol. 7, p. 618) et Hox 1-6 (Hox A 1) [23, 24] ont montré d'une manière spectaculaire l'implication de ces gènes dans l'organogenèse du cerveau postérieur et de la région branchiale. La mutation du gène Hox 1-5 produit des anomalies comparables à celles du syndrome de Di George qu'on peut mimer dans l'embryon de poulet en enlevant la crête neurale de la région rhombencéphalique. I a mutation du gène Hox 1-6 (Hox A1) produit, dans la même région, des anomalies portant essentiellement sur les dérivés neuraux des crêtes neurales rhombencéphaliques postérieures correspondant aux rhombomères 6,7 et
8. Enfin, l'expression spatiotemporelle du gène Hox 4-2 (Hox D4) peut être modifiée expérimentalement, s'il est placé sous le contrôle du promoteur du gène Hox 1-6 (Hox A1) qui s'exprime plus tôt et plus antérieurement. Les souris transgéniques hébergeant cette construction chimérique présentent des anomalies importantes du squelette cervical [25].

Ces résultats expérimentaux nous ont incités à reconsidérer les anomalies congénitales de l'homme affectant les régions du tronc cérébral, de la face et du cou.

A la lumière des travaux expérimentaux réalisés chez des embryons de poulet et de souris, on peut considérer qu'il existe aussi chez l'homme une segmentation originelle de la région cervico-faciale embryonnaire en métamères, ou unités branchiorhombomériques, que l'on ne distingue plus chez le fotus. Chaque unité est constituée de deux rhombomères consécutifs, du nerf qui en est issu et de l'arc branchial correspondant dont le mésenchyme est constitué par des cellules de la crête neurale céphalique du même niveau. Ainsi le premier arc branchial mandibulaire est innervé par le nerf trijumeau, provenant des rhombomères 2 et 3 , le deuxième arc branchial stylohyoïdien par le nerf facial, provenant des rhombomères 4 et 5 , les troisième et quatrième arcs (arcs thyrohyoïdien et crico-hyoïdien) sont innervés par les nerfs glossopharyngien et pneumogastrique, provenant respectivement des rhombomères $6-7$ et 7-8 (figure 5). Le rhombomère 8 comporte les motoneurones du nerf grand hypoglosse qui innerve la langue placée dans la partie ventrale de l'appareil branchial.

\section{Les malformations latérales de la face et du cou associées à des anomalies des nerfs crâniens et du tronc cérébral}

La segmentation branchiorhombomérique embryonnaire originelle éclaire considérablement l'analyse clinique des malformations cervico-faciales latérales du nouveauné. En effet, on peut montrer que 


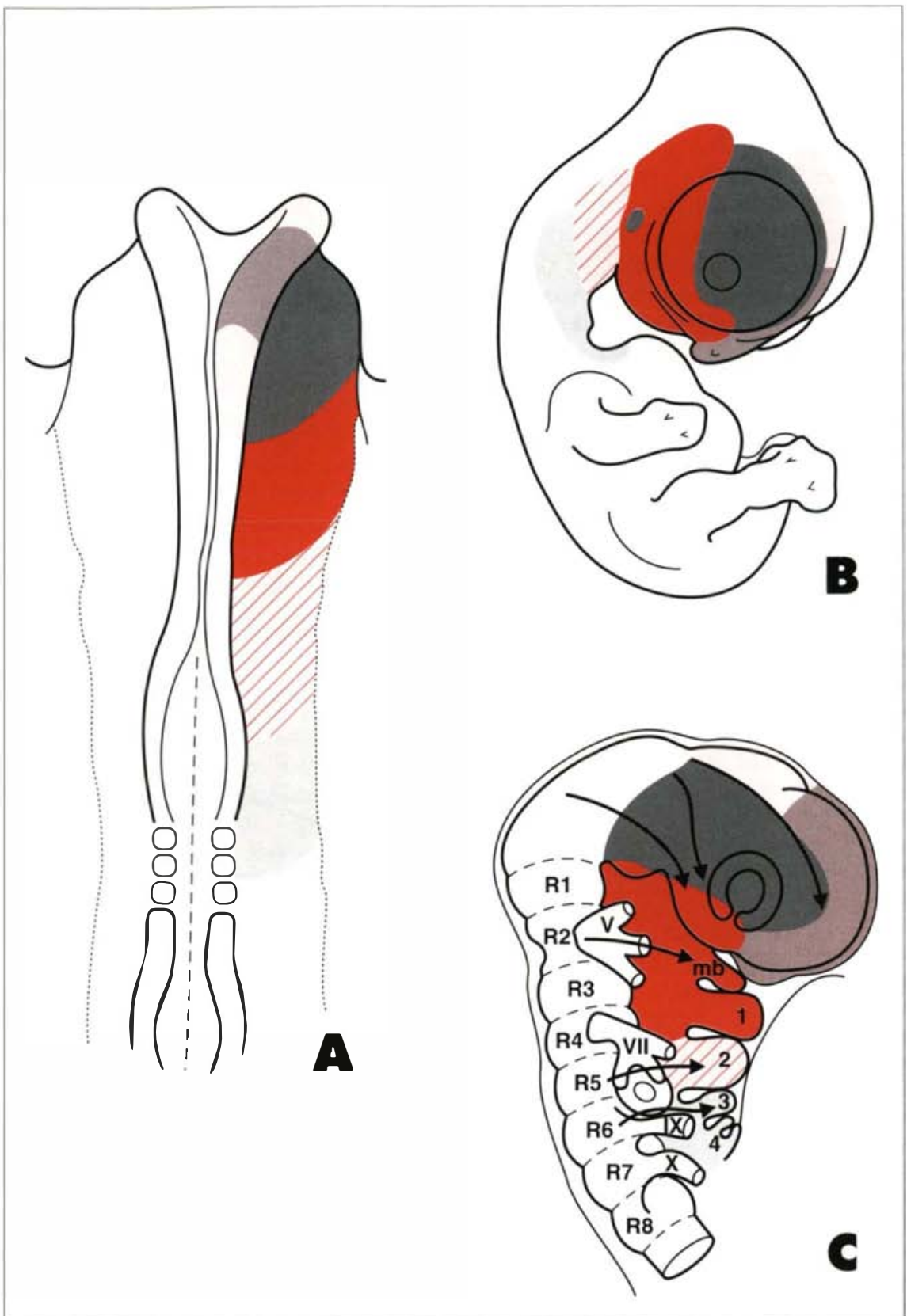

Figure 5. Les ectomères sont des unités branchiomériques : au stade de 3 somites (A) l'ectoderme de surface de l'embryon est représenté par des plages de couleurs différentes correspondant aux expériences réalisées [15]). En (B) représentation de l'évolution des bandes d'ectoderme ou ectomères - chez un embryon de 8 jours. En rouge, l'ectoderme du premier arc. En rose hachuré, l'ectoderme du deuxième arc. En gris clair, l'ectoderme des troisième et quatrième arcs. En (C) sont représentées les différentes unités branchio-rhombomériques.

- Aux rhombomères R2 et R3, où se forme le nerf trijumeau (nerf V), correspond le premier arc (1), en rouge $(m b=$ bourgeon maxillaire).

- Aux rhombomères R4 et R5 où se forme le nerf facial (nerf VII) correspond le deuxième arc (2), en rose hachuré.

- Aux rhombomères R6, R7 et R8 où se forment les nerfs glosso-pharyngien et pneumogastrique (nerf IX et $X$ respectivement) correspondent le troisième et le quatrième arc (3 et 4), en gris clair.

Les régions du bourrelet neural ectodermique colorées en rose foncé, bistre et rose clair (A) ont un devenir facial (B) (dans les fosses nasales, sur le bec supérieur et le crâne).

$\mathrm{m} / \mathrm{s} n^{\circ} 2$ vol. 10, frürier 94 l'origine des différentes malformations observées dans un syndrome donné correspond à une défaillance totale ou partielle du développement d'une unité segmentaire de la région du cerveau postérieur et des structures qui lui sont associées. Celles-ci concernent les cellules de la crête neurale d'un ou de plusieurs arcs branchiaux, ainsi que les nerfs dont les somas neuronaux sont situés dans les rhombomères correspondants. A chaque malformation faciale ou cervicale latérale, peut, en général, être associée une anomalie d'un nerf crânien issu du tronc cérébral, entraînant des troubles moteurs et sensitifs (parésie ou paralysie). Les anomalies du rhombencéphale lui-même consistent dans des hypoplasies ou des agénésies des structures qui en dérivent. Les plus fréquentes sont celles du cervelet, qui se manifestent de manière uni- ou bilatérale, et celles du tronc cérébral, qui sont caractérisées par des réductions volumétriques du tissu neural. Il est possible de mettre de telles malformations en évidence par la tomodensitométrie ou par la résonance magnétique nucléaire. Ainsi, dans une étude systématique du cerveau de 300 nourrissons malformés de la face, des malformations cérébrales situées au niveau du même métamère ont été mises en évidence dans les $13 \%$ des cas [26, 27].

En allant du rhombomère 2 au rhombomère 8 , on peut distinguer une série de syndromes dont les manifestations se situent au niveau d'unités segmentaires bien définies. Dans certains cas, cependant, ces syndromes sont associés, témoignant de la défaillance du développement de plusieurs niveaux rhombomériques consécutifs (figure 6).

\section{Les syndromes du premier arc}

Il s'agit de malformations par hypoplasie ou agénésie de certains dérivés de la crête neurale mésencéphalique et rhombencéphalique antérieure, constituant les bourgeons maxillaires et mandibulaires, associées à des anomalies motrices et sensitives du nerf trijumeau $\left(5^{e}\right.$ paire crânienne) issu des rhombomères 2 et 3 . Ces syndromes comportent parfois des paralysies totales 
ou partielles du nerf facial ( $7^{\mathrm{e}}$ paire crânienne) et une extension atteignant les muscles oculo-moteurs, dépendant pour leur innervation $\operatorname{des} 3^{e}, 4^{e}$ et $6^{e}$ paires de nerfs crâniens. Parmi les nombreuses variétés cliniques de ce syndrome, on peut distinguer chez l'homme:

- Le syndrome de Goldenhar comportant des malformations externes des paupières, du pavillon de l'oreille, des dermoïdes situés sur la partie externe de la conjonctive et des petits fibrochondromes jugaux préauriculaires.

- Le syndrome de Franceschetti ou de Treacher Collins (encore appelé dysostose mandibulo-faciale), dans lequel sont associées des agénésies du squelette latéral de la face, affectant l'os malaire, l'arcade zygomatique, le tympanal, le condyle et les branches montantes mandibulaires. - Le syndrome oto-mandibulaire qui comporte une agénésie partielle ou totale d'une hémi-mandibule, du marteau, de l'enclume et une hypoplasie plus ou moins importante de la partie supérieure du pavillon de l'oreille qui dérive du premier arc branchial. Dans cette malformation, sont absents le conduit auditif et la caisse du tympan qui dérivent de la première poche branchiale, ainsi que le tympan (un dérivé de la première fente branchiale).

Une étude systématique a été réalisée sur vingt-deux enfants qui présentaient à la naissance des malformations apparentes du premier arc branchial et de ses dérivés [28]. Dans $36 \%$ des cas, des malformations du tronc cérébral, et, dans $68 \%$ des cas, des anomalies des nerfs correspondants y étaient associées. Les anomalies du tronc cérébral consistaient en des malformations de la fosse postérieure et de la protubérance annulaire, des méga-grandes citernes et des kystes dermoïdes du cervelet. Les anomalies nerveuses consistaient en des paralysies du trijumeau, du nerf facial et du nerf moteur oculaire externe. Il faut ajouter que dans ce groupe de nourrissons malformés, $18 \%$ des enfants présentaient des microphtalmies, témoignant de la défaillance de la crête neurale du même niveau dans la genèse de la sclérotique et de la choroïde.
Ces anomalies, associant des malformations latérales de la face et du tronc cérébral, ont été déjà rapportées par Aleksic et al. [29] qui signalent la grande fréquence des paralysies oculomotrices dans les syndromes de Franceschetti et de Goldenhar et rapportent une observation exemplaire de malformation du premier arc avec fente macrostomique associée à une anophtalmie, un lipome du cervelet et une agénésie nucléaire des nerfs crâniens des rhombomères 2,3 et 4 .

\section{Les syndromes du deuxième arc}

Ce sont des malformations par agénésie ou hypoplasie de certains dérivés de la crête neurale rhombencéphalique constituant le deuxième arc branchial innervé par le nerf facial (rhombomères 4 et 5 ). Les anomalies cervico-faciales de ces syndromes sont moins spectaculaires que celles des syndromes du premier arc. Elles n'en demeurent pas moins significatives. Ces syndromes se manifestent par une absence ou une hypoplasie du lobule de l'oreille qui dérive du deuxième arc, des aplasies du derme de la partie moyenne du cou, avec parfois du tissu thymique ectopique, et des fistules ectodermiques du sinus cervical dérivé de la deuxième poche ectobranchiale ou des kystes amygdaloïdes de la deuxième poche endobranchiale. Des malformations du corps et de la petite corne de l'os hyoïde qui dérivent du deuxième arc sont aussi observées. A ces anomalies s'associe parfois une paralysie complète des nerfs faciaux et des nerfs moteurs oculaires externes. Cliniquement, les nourrissons ne présentent aucune mimique faciale. Ils sont atteints, par ailleurs, d'un strabisme interne bilatéral. L'ensemble de ces anomalies constitue le syndrome de Moebius (figure 6).

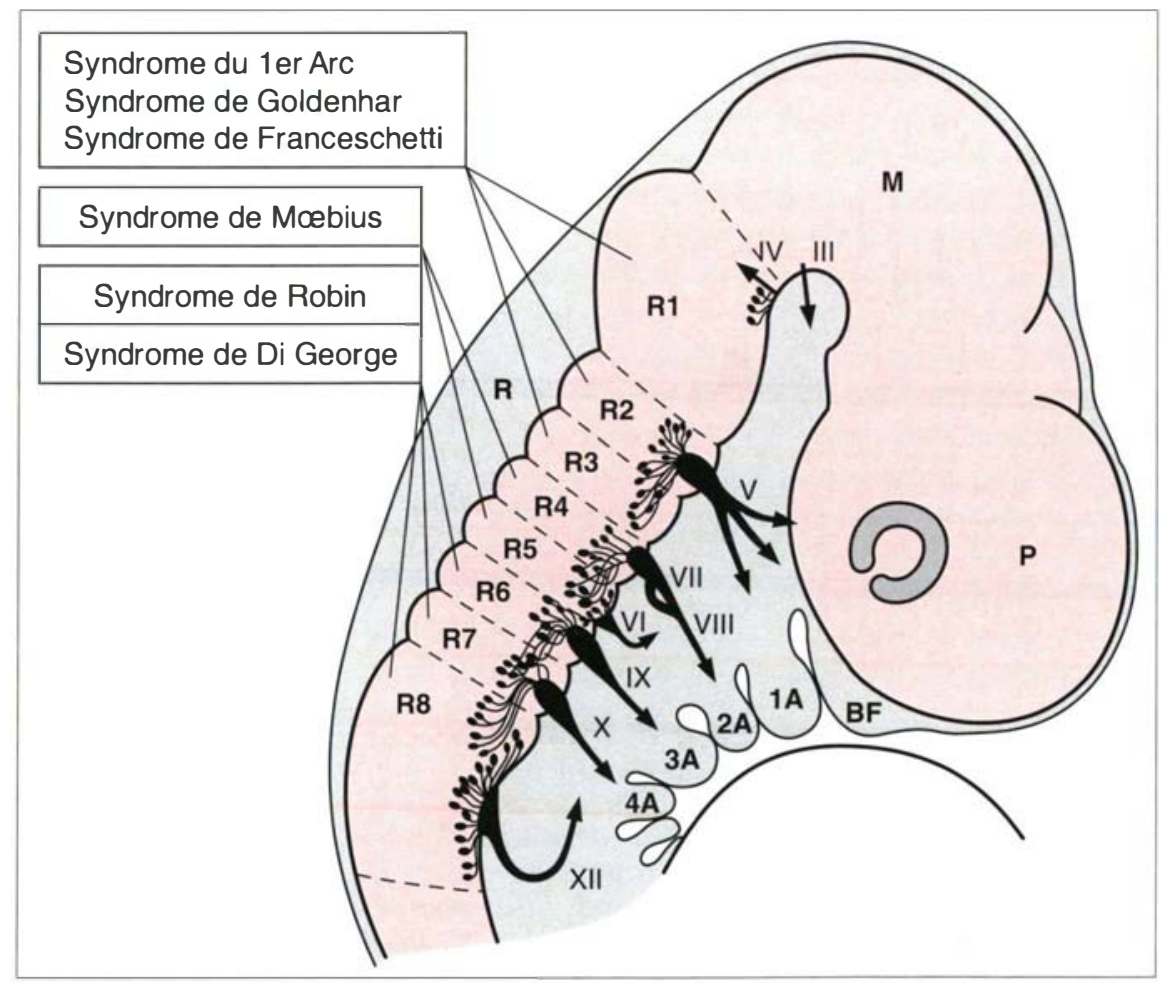

Figure 6. Schéma des huit rhombomères du tronc cérébral (R1, R8) au niveau desquels sont représentés les corps cellulaires d'origine des nerfs branchiaux (V: trijumeau; VII et VIII: facial et acoustique; IX: glossopharyngien; $X$ : pneumogastrique; III, IV et VI: nerfs somatiques de l'œil; XII: nerf somatique de la langue). Les divers syndromes malformatifs latéraux de la face sont mentionnés en corrélation avec les niveaux rhombomériques des nerfs et les niveaux d'origine de la crête neurale migrant dans les arcs branchiaux. $P$ : prosencéphale; $M$ : mésencéphale; $R$ : rhombencéphale; $B F$ : bourgeon frontal; $1 A, 2 A, 3 A$ et $4 A$ : les quatre premiers arcs branchiaux. 


\section{Les syndromes des troisièmes et quatrièmes arcs branchiaux}

Ils sont la conséquence du défaut de développement des cellules de la crête neurale rhombencéphalique postérieure dans les régions cervicale, latérale et thoracique supérieure, correspondant aux rhombomères 6,7 et 8. Les malformations morphologiques affectent le thymus, les parathyroïdes, les cellules à calcitonine et les dérivés des arcs aortiques ainsi que le tronc aorto-pulmonaire issu du cœur. Ces malformations sont en règle générale associées à des anomalies motrices des nerfs pneumogastriques $\left(10^{\mathrm{e}}\right.$ paire crânienne) et glossopharyngiens (9e paire crânienne) dont les corps cellulaires sont dans les rhombomères 6 , 7 et 8 . Les deux syndromes les plus marquants sont ceux de Di George et de Robin [30, 31] (figure 6).

Le syndrome de Di George ([30], $m / s \quad n^{\circ} 2$, vol. $\left.8, \quad p .182\right)$ comporte une hypoplasie ou une agénésie du thymus et des parathyroïdes, une anomalie des troisième, quatrième et sixième arcs aortiques et des malformations cardiaques cono-troncales et septales inter-auriculaires. Ce syndrome peut être reproduit expérimentalement par l'ablation de la crête neurale rhombencéphalique postérieure montrant le rôle essentiel joué par cette structure dans l'organogenèse de la région hypobranchiale inférieure et intrathoracique [13, 32]. L'utilisation des chimères embryonnaires entre caille et poulet a en effet montré que la crête neurale rhombencéphalique postérieure fournit les constituants mésenchymateux du thymus, des parathyroïdes, ainsi que la paroi musculo-aponévrotique des artères dérivées des arcs aortiques [13]. Le tronc artériel du cour reçoit aussi une large contribution des crêtes neurales issues des niveaux rhombomériques 6,7 et 8 [32].

La mutation du gène $H o x A 3$ chez la souris [22] reproduit très fidèlement le syndrome de Di George. Chisaka et Capecchi ont créé des embryons de souris homozygotes pour l'interruption du gène Hox 1 -5 (Hox A3) par recombinaison homologue $\left(m / s n^{\circ} 6\right.$, vol. $7, p$. 618). I.es souriceaux atteints meurent à la naissance ou dans les heures qui sui-

$\mathrm{m} / \mathrm{s} n^{\circ} 2$ vol. 10, frurrier 94 vent du fait de malformations du coeur ou des voies aériennes supérieures. Le syndrome malformatif des souris déficientes de la fonction Hox 1-5 ressemble au syndrome de Di George de l'homme. Cela ne signifie pas que ce dernier soit la conséquence directe d'une mutation homozygote du gène $\mathrm{HOX} 1-5$ chez l'homme. Le syndrome de Di George est en effet autosomique dominant alors que la mutation murine est récessive. Chez l'homme, certains syndromes de Di George sont associés à des micro-délétions chromosomiques affectant le chromosome 22 alors que le gène Hox 1-5 est localisé sur le chromosome 7. Ainsi le gène Hox $1-5$ et le gène atteint dans le syndrome de $\mathrm{Di}$ George pourraient-ils intervenir sur une même cascade d'événements régulateurs $\left(m / s n^{\circ} 6\right.$, vol. $7, p$. 618). Le syndrome de Pierre Robin [31] a pour expression clinique la défaillance du fonctionnement du tronc cérébral correspondant aux rhombomères 6,7 et 8 , c'est-à-dire les défaillances précoces de l'organisation des fonctions orales et digestives céphaliques (succion et déglutition), de la ventilation (commande et régulation) et de la régulation parasympathique du cour. Le tableau clinique comporte aussi une division vélopalatine avec un rétrognathisme engendré par la position nasale de la langue, témoin de la défaillance fotale de l'organisation neurophysiologique du tronc cérébral au niveau des rhombomères 6,7 et 8 . Dans $25 \%$ des cas, une malformation conotroncale du coeur est constatée, ce qui peut être interprété comme une association des syndromes de Di George et de Robin.

L'inactivation par recombinaison homologue dans les cellules souches de souris du gène Hox 1-6 (Hox A1) $[23,24]$ engendre des sujets homozygotes létaux dont les anomalies sont situées dans la partie postérieure du cerveau. Les structures affectées par la mutation dérivent d'un territoire correspondant aux rhombomères 4 , 5 , 6 et 7 . Les souriceaux meurent d'anoxie à la naissance, situation homologue, semble-t-il, à celle du syndrome de Robin. La taille des ganglions sensitifs des nerfs auditif, glossopharyngien et pneumogastri- que, est très réduite chez le souriceau. De même, le noyau moteur du nerf facial est absent. Ainsi, seules les cellules des crêtes neurales destinées aux ganglions des nerfs crâniens paraissent être affectées par l'inactivation du gène Hox 1-6. Deux gènes différents du groupe $H o x$ A, exprimés dans un même territoire du rhombencéphale, paraissent donc être impliqués dans le devenir des cellules des crêtes neurales vers une destinée, soit neurale pour Hox AI (ganglions nerveux de la partie basse du tronc cérébral), soit mésenchymateuse pour Hox A3 (dérivés des arcs branchiaux). Cette situation génétique pourrait apporter une explication aux deux syndromes apparentés de Robin et Di George, le premier ayant une dominante nerveuse, le second ayant une dominante organique branchiale.

\section{Le syndrome de dysfonctionnement néonatal du tronc cérébral [33]}

L'intérêt de rassembler l'ensemble de ces syndromes sous un même et unique mécanisme neurodysembryologique est de montrer qu'ils ont en commun un tableau clinique néonatal, identifiable à celui appelé syndrome de dysfonctionnement néonatal du tronc cérébral, dont le gradient de gravité croissant va du rhombomère le plus rostral au rhombomère le plus distal. Ce syndrome associe des signes cliniques de dysfonctionnement ou de défaillance des fonctions dont la localisation neuroanatomique est le tronc cérébral, c'est-à-dire la succion déglutition et la motricité oesophagienne, la commande et la régulation de la ventilation, la régulation parasympathique du cœur. L'ensemble de ces symptômes est aggravé pendant le sommeil, minoré par l'éveil.

\section{Les associations syndromologiques}

Elles sont fréquentes et correspondent au développement défaillant de plusieurs unités branchiorhombomériques cervico-faciales. Leurs constatations pourraient témoigner, soit de l'origine polyclonale des somas des neurones entrant dans la constitution des noyaux des nerfs crâniens issus du tronc cérébral avant la détermination des frontières entre les 


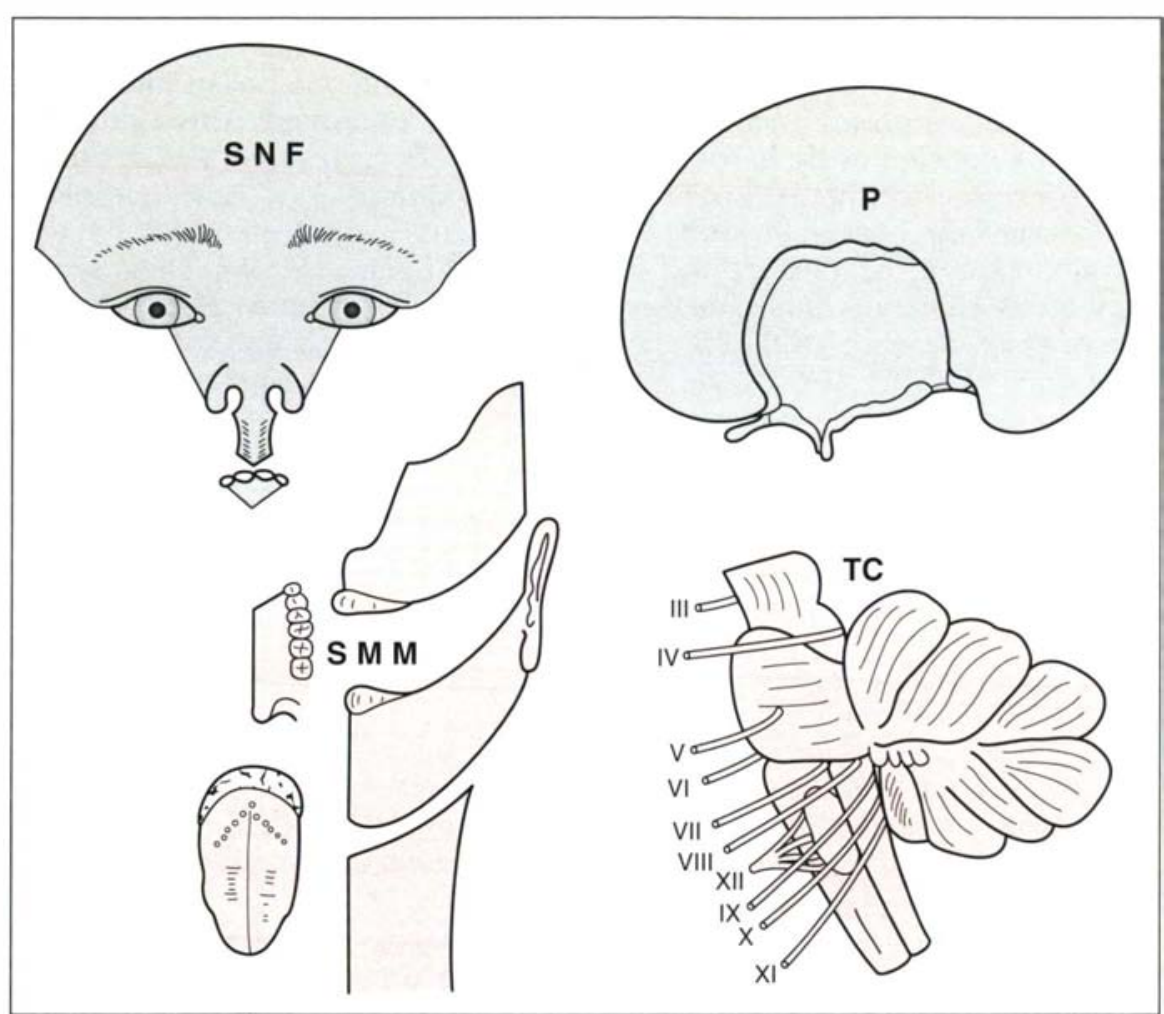

Figure 7. Grille de lecture des malformations associées de la face et du cerveau. Aux structures naso-frontales (SNF) correspond le prosencéphale $(P)$ et aux structures maxillo-mandibulaires (SMM) et cervicales correspondent le tronc cérébral (TC) et les nerfs crâniens.

rhombomères, soit de l'existence d'atteinte simultanée de plusieurs rhombomères. L'association syndromologique constatée concerne deux, parfois trois rhombomères adjacents, mais jamais de rhombomères disjoints. Les associations les plus typiques en clinique humaine concernent les syndromes de Robin et de Di George [30, 31], les syndromes de Robin et de Moebius [31], les syndromes du premier arc et de Moebius, les syndromes de Robin, du premier arc et de Moebius.

Ces associations sont gravissimes sur le plan clinique car le syndrome de dysfonctionnement du tronc cérébral est complet et responsable d'un pronostic très péjoratif.

\section{Conclusion : la face, structure prédictive du cerveau}

La segmentation originelle branchiorhombomérique permet d'établir une grille de lecture et d'analyse clinique des malformations (figure 7).

\section{TIRÉS A PART}

\section{N.M. Le Douarin.}

\section{Summary}

Rhombomeres, Hox code neural crest and facial malformations

The transient segmentation of the developing hindbrain into rhombomeres, already described at the turn of the century, has recently been confirmed and further documented at the cellular and molecular levels. Cell tracing experiments carried out in the chick embryo have demonstrated the polyclonal origin of the neurons within each rhombomere and the absence of cell mixing between adjacent rhombomeres after interrhombomeric boundaries are established. Each hindbrain segment is characterized during ontogeny by the transient expression of a definite combinatoire of $\mathrm{Hox}$ genes defining a Hox code. Experimental alteration of this code at the critical stages where $H o x$ genes act as selectors of a particular developmental programme, results in malformations of a particular branchiorhombomeric compartment. Studies based on the use of the quail-chick marker system in the avian embryo have revealed that the facial and hypobranchial structures are derived from the neural crest. The cells originating from the neural crest express the same Hox code than those of the corresponding rhombomeres. Malformations of the facial and hypobranchial regions are relatively common in man. They are interpreted here in the light of the recent discoveries concerning the metamerisation of the branchiorhombomeric area in the embryo and its control by selector genes of the Hox complex. We argue that each described syndrome results from developmental abnormalities of one or two consecutive hindbrain segments. As a consequence, facial abnormalities have a predictive value concerning the development of the hindbrain itself. Facial structures being neural crest-derived, constitute a « marker " of neurogenesis in the transverse levels of the brain from which they arise. 